\title{
Android APK Identification using Non Neural Network and Neural Network Classifier
}

\author{
Djarot Hindarto*, Handri Santoso \\ Magister Technology Informatic, Pradita University \\ Tangerang, Banten, Indonesia \\ Email: djarot.hindarto@student.pradita.ac.id, handri.santoso@student.pradita.ac.id
}

*Corresponding Author

\begin{abstract}
The purpose of this study is to identify Android APK files by classifying them using Artificial Neural Network (ANN) and Non Neural Network (NNN). The ANN is Multi-Layer Perceptron Classifier (MLPC), while the NNN are KNN, SVM, Decision Tree, Logistic Regression and Naïve Bayes methods. The results show that the performance using NNN has decreasing accuracy when training using larger datasets. The use of the K-Nearest Neighbor algorithm with a dataset of 600 APKs achieves an accuracy of $91.2 \%$ and dataset of 14170 APKs achieves an accuracy of $88 \%$. The using of the Support Vector Machine algorithm with the 600 APK dataset has an accuracy of $99.1 \%$ and the 14170 APK dataset has an accuracy of $90.5 \%$. The using of the Decision Tree algorithm with the 600 APK dataset has an accuracy of $99.2 \%$, the 14170 APK dataset has an accuracy of $90.8 \%$. The experiment using the Multi-Layer Perceptron Classifier has increasing with the $600 \mathrm{APK}$ dataset reaching $99 \%$, the 7000 APK dataset reaching $100 \%$ and the 14170 APK dataset reaching $100 \%$.
\end{abstract}

Key words: Multi Layer Perceptron Classifier, Artificial Neural Network, Non Neural Network, APK Malware Android

\section{INTRODUCTION}

Currently, the development of APK malware is increasing, along with the number of Package Kit Applications (APKs) which are applications that run on the Android operating system. So many Androids APKs, causing more and more certain parties to attack for purposes that are profitable for malware makers. Therefore, there are many losses for Android Mobile phones that have been infected by malware. From year to year the development of malware has increased, for this reason this research uses the topic of Android malware. Intents are interfaces that connect interactions between Activities in an Android APK. In addition, Intents send data to other Activities, such as sending data to other applications (Gmail, Google Map, etc.). In essence, Intent is a mechanism to perform an action and communicate between application components.

Originality: Most of the journals in the literature review focus on feature permissions, rarely exploring feature intents. An Android APK to activate an action or activity calls a component, sends data, requires a feature intent. Without feature intents, Android cannot perform action functions. Therefore, this research focuses on feature permissions and feature intents. Malware classification has been carried out by applying machine learning, such as the use of the K-Nearest Neighbor algorithm, Support Vector Machine and Decision Tree. The average classification results are good, but if you use a large dataset, the classification performance decreases. Then the experiment was carried out by applying a deep learning algorithm, namely Multi-Layer Perceptron. Some experimental results continue to increase in accuracy with the increasing number of datasets.

The aim of this study is to identify Android APK files by classifying Android APK files using the Multi-Layer Perceptron Classifier. The main contribution of this paper is to improve the accuracy of malware classification performance by applying the Multi-Layer Perceptron Classifier algorithm.

Some research questions in this study:

RQ 1, How to extract malware dataset using permission feature and intent feature?

RQ 2, What is the percentage of application of the K-NN algorithm, Support Vector Machine and Decision Tree?

RQ 3, What is the percent increase in accuracy with the implementation of the Multi-Layer Perceptron algorithm? RQ 4, Is it effective to perform malware analysis using static methods?

This article is organized as follows: Section 2 presents related work on several articles related to the Classification of Android malware. Section 3 describes the methodology used. Section 4 describe Propose Method. Section 5 presents the results of the experiments that have been carried out. Section 6 includes a summary of the paper.

\section{RELATED WORK}

In this study, we compare with previous research that discusses the Android malware APK. The attackers created malware using a new method of targeting victims of Android mobile phones. Several studies have used effective tools to carry out the malware detection process as accurately as possible.

Table I shows a lot of research using extract on feature permissions, system calls, API Calls, Net Info, but still very rarely uses feature intent. This feature intent is an addition to the research, in addition to using feature permissions. This research uses feature permission and feature intent. 
TABEL I. LITERATURE REVIEW

\begin{tabular}{|c|c|c|c|c|}
\hline Work & Features & Classifier & Data & Performance \\
\hline \multirow[t]{6}{*}{ [1] } & \multirow[t]{6}{*}{ Permission } & \multirow[t]{3}{*}{ SVM } & 10000 & $\begin{array}{l}\text { Precision } \\
98.20 \% \\
\text { Recall } 95.80 \% \\
\text { F-measure } \\
96.96 \%\end{array}$ \\
\hline & & & 25000 & $\begin{array}{l}\text { Precision } \\
97.16 \% \\
\text { Recall } 93.75 \% \\
\text { F-measure } \\
95.42 \%\end{array}$ \\
\hline & & & 60000 & $\begin{array}{l}\text { Precision } \\
95.17 \% \\
\text { Recall } 92.86 \% \\
\text { F-measure } \\
94.00 \%\end{array}$ \\
\hline & & \multirow[t]{3}{*}{ DT } & 10000 & $\begin{array}{l}\text { Precision } \\
98.99 \% \\
\text { Recall } 96.10 \% \\
\text { F-measure } \\
97.53 \%\end{array}$ \\
\hline & & & 25000 & $\begin{array}{l}\text { Precision } \\
96.10 \% \\
\text { Recall } 93.20 \% \\
\text { F-measure } \\
94.68 \%\end{array}$ \\
\hline & & & 60000 & $\begin{array}{l}\text { Precision } \\
92.11 \% \\
\text { Recall } 91.10 \% \\
\text { F-measure } \\
91.60 \%\end{array}$ \\
\hline \multirow[t]{6}{*}{ [2]. } & \multirow{6}{*}{$\begin{array}{l}\text { Application } \\
\text { Programmi } \\
\text { ng Interface } \\
\text { (API) calls, } \\
\text { Permissions }\end{array}$} & SVM & $\begin{array}{c}347 \\
\text { benign, } \\
365 \\
\text { mal }\end{array}$ & $96.2 \%$ \\
\hline & & $\mathrm{KNN}$ & $\begin{array}{c}347 \\
\text { benign, } \\
365 \\
\text { mal }\end{array}$ & $97.2 \%$ \\
\hline & & DT & $\begin{array}{c}347 \\
\text { benign, } \\
365 \\
\text { mal }\end{array}$ & $96.6 \%$ \\
\hline & & $\mathrm{RF}$ & $\begin{array}{c}347 \\
\text { benign, } \\
365 \\
\text { mal }\end{array}$ & $97.8 \%$ \\
\hline & & $\begin{array}{l}\text { Naïve } \\
\text { Bayes }\end{array}$ & $\begin{array}{c}347 \\
\text { benign, } \\
365 \\
\text { mal }\end{array}$ & $93.9 \%$ \\
\hline & & GRU & $\begin{array}{c}347 \\
\text { benign, } \\
365 \\
\text { mal }\end{array}$ & $98.2 \%$ \\
\hline [3] & Permission & $\begin{array}{c}\text { Chi-Square } \\
\text { \& Naïve } \\
\text { Bayes }\end{array}$ & $\begin{array}{c}5000 \\
\text { mal } \\
\text { Drebin } \\
\text { and } \\
5000 \\
\text { benign } \\
\text { (Andro } \\
\text { zo) }\end{array}$ & $91.1 \%$ \\
\hline [4] & $\begin{array}{c}\text { API \& } \\
\text { Permissions }\end{array}$ & $\begin{array}{c}\text { RF } \\
\text { ANN }\end{array}$ & $\begin{array}{c}5000 \\
\text { benign, } \\
1260 \\
\text { mal }\end{array}$ & $\begin{array}{l}94 \% \\
94 \%\end{array}$ \\
\hline
\end{tabular}

\begin{tabular}{|c|c|c|c|c|}
\hline Work & Features & Classifier & Data & Performance \\
\hline [5] & $\begin{array}{c}\text { Permissions } \\
\text {, APIs }\end{array}$ & SVM & $\begin{array}{c}1500 \\
\text { benign, } \\
1500 \\
\text { mal }\end{array}$ & $99.6 \%$ \\
\hline [6] & $\begin{array}{l}\text { APIs, Net } \\
\text { Info, etc. }\end{array}$ & $\begin{array}{c}\text { SVM } \\
\text { K-NN ERT }\end{array}$ & $\begin{array}{c}5560 \\
\text { benign, } \\
5560 \\
\text { mal }\end{array}$ & $\begin{array}{l}90.4 \% \\
90.47 \% \\
93.66 \%\end{array}$ \\
\hline [7] & $\begin{array}{l}\text { APIs, Net } \\
\text { Info }\end{array}$ & Ensemble & $\begin{array}{c}4403 \\
\text { benign, } \\
3982 \\
\text { mal } \\
\end{array}$ & $99.7 \%$ \\
\hline [8] & $\begin{array}{l}\text { APIs, Net } \\
\text { Info }\end{array}$ & $\begin{array}{c}\text { NB } \\
\text { RF } \\
\text { K-NN } \\
\text { XGBOOST } \\
\text { DL }\end{array}$ & $\begin{array}{c}11187 \\
\text { benign, } \\
18677 \\
\text { mal }\end{array}$ & $\begin{array}{l}87 \% \\
96 \% \\
94 \% \\
97 \% \\
96 \% \\
\end{array}$ \\
\hline [9] & $\begin{array}{l}\text { Permissions } \\
\& \text { Intents }\end{array}$ & $\begin{array}{c}\text { BN } \\
\text { SVM } \\
\text { DT } \\
\text { LR } \\
\end{array}$ & $\begin{array}{c}1846 \\
\text { benign, } \\
5560 \\
\text { mal } \\
\end{array}$ & $\begin{array}{c}95.5 \% \\
94 \% \\
83 \% \\
91 \% \\
\end{array}$ \\
\hline [10] & $\begin{array}{l}\text { Permissions } \\
\text { \& Intents }\end{array}$ & Ensemble & $\begin{array}{c}445 \\
\text { benign, } \\
1246 \\
\text { mal }\end{array}$ & $99.8 \%$ \\
\hline
\end{tabular}

\section{Metodology}

In this section, the researcher discusses malware analysis and classification [11] research methodology. Performing malware analysis there are three analyzes, namely static analysis[12], dynamic analysis and hybrid analysis. The use of the malware identification or detection method is a supervised learning classification. The algorithm used is KNN, Support Vector Machine and Decision Tree, as well as Deep Learning Multi-Layer Perceptron Classifier[13] [14].

\section{A. Static Analysis}

Static analysis [15] is a malware analysis method by analyzing source code. Reverse engineering is used to obtain the source code file, which converts the executable file into a source code file. To analyze the malware APK file, for example, the APK file must be reverse engineered. Analyzing static malware does not need to run the application. Using the JADX module from APKTOOL, to do reverse engineering. The source code to be analyzed is the AndroidManifest.xml file. This file is then read or parse android-permission and android-intent.

Some purposes for reverse engineering:

- To know the protocol of a program. For example: want to create a command line Instagram client.

- To find out the API used by a program. For example, you want to know how to turn on the camera flash as a flashlight.

- To find security bugs for a program.

- To find out if a program violates copyright. For example, we suspect that a program uses a commercial library that we created, without paying for a license.

- For forensic purposes. For example, we want to know the data format used by a program. 


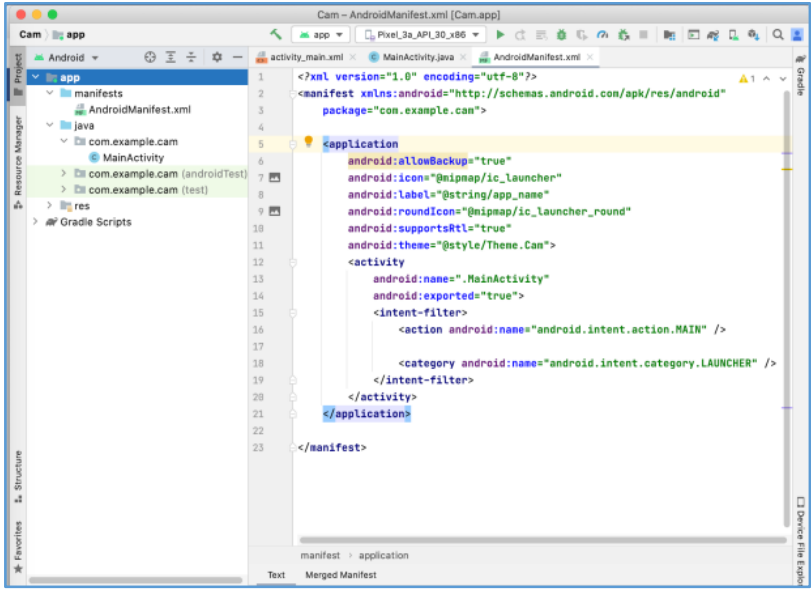

Fig. 1. Architecture Multi-Layer Perceptron

Figure 1, AndroidManifest.xml file, the result of the reverse engineering process. This file will parse the permissions and intent features.

\section{B. Dynamic Analysis}

Malware is a threat to Android, various methods are used to analyze malware, one of which is using dynamic analysis. Analyzing Android malware with dynamic methods aims to understand its behavior and improve the ability to detect it. Dynamic analysis also takes an analytical approach to analyze Android malware behavior. How to perform analysis by running malware code in a virtual environment to understand the actual behavior of malware. The dynamic analysis method does not examine the source code, but runs the malware files in a controlled environment, which is called a sandbox. This way the behavior of the malware can be analyzed in a controlled environment, this is very useful where the malware does not spread to other systems. After observing the behavior of malware, a log of malware activity is obtained. This log will be analyzed.

\section{Hybrid Analysis}

Dynamic malware analysis is a combination of static analysis and dynamic analysis, where the analysis runs the malware in a controlled environment after that it also analyzes the source code. Hybrid model analysis is a perfect and complete analysis for analyzing a malware.

\section{K-Nearest Neighbor}

K-Nearest Neighbor (KNN) [16] [17] is a classification algorithm using a way to measure the distance, which is measured from the $\mathrm{k}$ nearest neighbors. This classification projects the training dataset in a multidimensional space. The space is divided into sections that describe the character of the data. Each training data is represented as points in a multidimensional space. Where the KNN classification[18] [19] process is looking for the point $\mathrm{c}$ closest to the new (c). The general formula is to find the Euclidean distance, Hamming distance, Manhattan distance, and Minkowski Distance.
Euclidean distance [20] is a formula for finding the distance between two points in two-dimensional space. Hamming distance [21] is a way to find the distance between two points which is calculated by the length of the binary vector formed by the two points in the binary code block. Manhattan Distance [22] is a formula to find the distance $\mathrm{d}$ between 2 vectors in $\mathrm{n}$ dimensional space. Minkowski distance is a formula for measuring between two points in a normal vector space which is a hybridization that generalizes the Euclidean distance and Manhattan distance. The K-Nearest Neighbor (KNN) [23] [24] algorithm is a classification of objects based on the learning data that is closest to the object. Then the determination of the $\mathrm{K}$ value is carried out. It is determined that the $\mathrm{K}$ value is odd, after that a vote is carried out on the closest distance. Advantages of KNN (K-Nearest Neighbor), dataset used for training is very nonlinear and easy to implementation. Disadvantages of KNN (KNearest Neighbor): Need to indicate the parameter $\mathrm{K}$ (number of nearest neighbors). Does not handle missing values implicitly. Sensitive to data outliers (outliers). Vulnerable to non-informative variables. Vulnerable to high dimensionality. The computational cost is quite high, because it is necessary to calculate the distance from each testing data to the entire training data.

\section{E. Support Vector Machine}

Support Vector Machine (SVM) was first presented by Boser, Guyon and Vapnik in 1992. Support Vector Machine is a supported classification algorithm by finding the hyperplane with the largest margin. There are three main sections in SVM, namely Supervised, Classified and Hyperplane with the largest margin. How the Support Vector Machine [25] [26] [27] works. Support vectors are two closely spaced data that come from different classes or groups, these two data will be used as support vectors. Hyperplane [28] [29] is the dividing line between support vectors. Max Margin[30] is the distance between the support vector and the hyperplane, the margin distance must be maximum to be able to anticipate the similarity of one data to another. For non-linear data, SVM Kernel Trick [31] [32] [33] is used by creating new dimensions. So that it can create a hyperplane. The advantage of SVM is that Supervised is able to control the accuracy of classification and Kernel trick is able to classify with nonlinear data. Disadvantages of SVM, not good for large amounts of data and Kernel trick is not easy to implement.

\section{F. Decision Tree}

The Decision Tree [34] [35] algorithm was developed by J. Ross Quinlan, in 1975. Decision tree is a popular classification method, because it is easy to interpret. Predictive model that uses a tree structure. Another term for Decision Tree is Classification and Regression Tree (CART)[35] [36] [37] which is a decision tree. Decision trees can convert data into decision trees and decision rules. The benefits of DT are its ability to break down complex decision-making processes into simpler ones, so that decision-makers better interpret problem solutions. 
Making a Decision Tree model[38] [39] is like drawing an inverted tree where the Root Node is in the top position. Internal Node that has 1 input and at least 2 outputs. Leaf Node is the final Node, has 1 input and has no output.

\section{G. Multi-Layer Perceptron Classifier}

Multi-Layer Perceptron is a classification algorithm that works by using a deep neural network. This algorithm is very different from machine learning algorithms based on statistical science. By using the deep neural network method, it is expected that the performance of the model is more accurate, when compared to machine learning. Figure 2 is the architecture of the Multi-Layer Perceptron Classifier to complete the classification of the malware dataset.

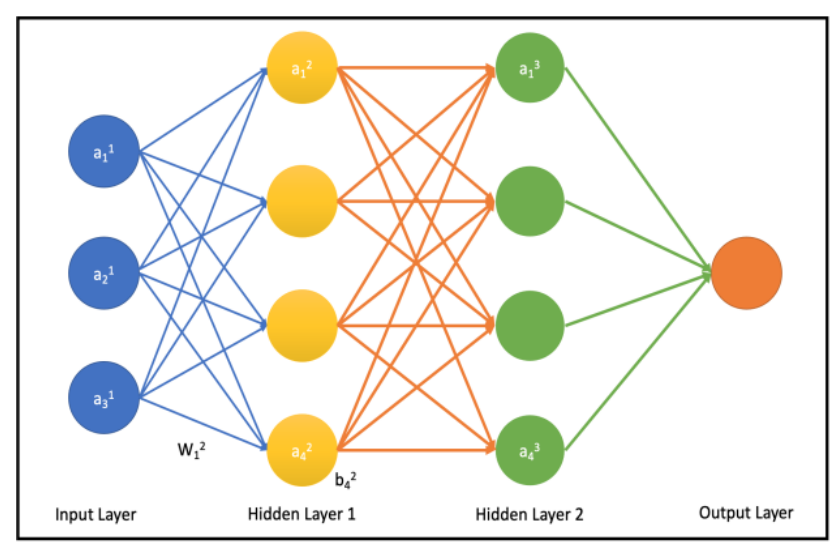

Fig. 2. Architecture Multi-Layer Perceptron

\section{Proposed Method}

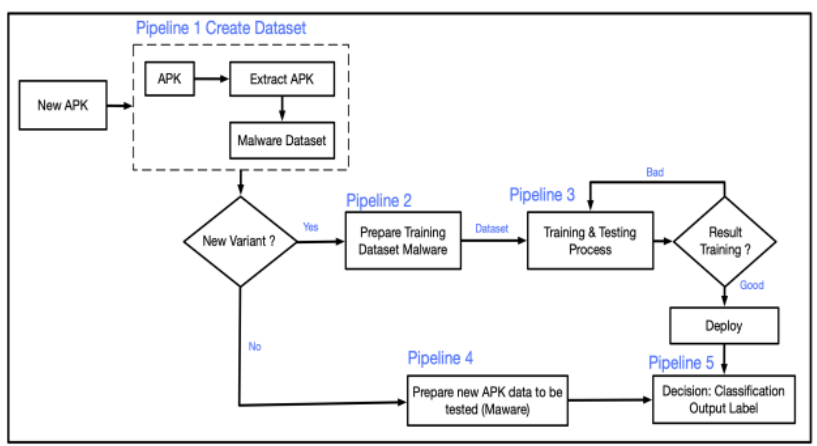

Fig. 3. Architecture Multi-Layer Perceptron

\section{A. Pipeline 1: Create Dataset}

This stage is to create a dataset from Android APK files that are indicated as malware or Benign. The malware APK files are downloaded from the University of New Brunswick. The file has been labeled for types of malwares. The downloaded file is accommodated to local storage, then the classification process is carried out and stored in a similar folder. Next, the Android APK file extraction feature is carried out using reverse engineering. Many reverse engineering tools are commonly used. In this research, reverse engineering uses the JADX module. The result of the reverse engineering process is some folders and files AndroidManifest.xml. Files and folders other than AndroidManifest.xml are deleted, while
AndroidManifest.xml is then parsed to read the permissions and intent features. The results of the feature extraction process produce a malware dataset. The next process is classification using machine learning or deep learning algorithms.

\section{B. Pipeline 2: Prepare Training Dataset malware}

Before training the malware dataset, the prepare stage is very necessary. To generate a model from a machine learning or deep learning training process must use a clean dataset, a good dataset (no null, incorrect data in features). The dataset must ensure that the contents of the malware Dataset should not be mixed with the Benign data. If there is a mixture of malware and Benign, the resulting model will experience errors and affect the performance of the model. In addition to the data cleaning process, there are also engineering features, namely feature analysis and the most influential features. This process must be carried out because this process is also very influential on the resulting model. The next process is to create a uniform dataset, in the sense that if there are five groups of datasets, then the dataset must be an unmixed dataset. For example, the malware APK dataset is of the Ransomware type, then the Ransomware dataset should not be mixed with the Riskware APK dataset.

The division of the number of datasets for machine learning is to divide the $70 \%$ training dataset and $30 \%$ testing data. But there is no requirement to do so. There are also those who share it, $60 \%$ training data and $40 \%$ testing data. Sharing datasets for deep learning, training data, validation data and testing data. Example (Data Training + Data Validation) $=70 \%$, while testing data is $30 \%$. Cross validation of datasets or swapping training positions with testing is also carried out to get the performance model that will be generated by machine learning or deep learning.

Some of the reasons for this data preparation is done: 1) The data owned is not ideal, there is data that is missing value. Missing data in the dataset will result in a declining model for its performance. Filling must be done so that the dataset becomes intact and good. It is not permissible to fill in the dataset arbitrarily and an analysis of the features or dimensions of the appropriate dataset must be carried out.

2) There are different data formats. To avoid differences in formats in the feature dataset, it is necessary to check, validate the dataset and analyze the features of the dataset.

3) Small dataset or imbalanced dataset from ideal in terms of quantity. Small datasets are not ideal for machine learning or deep learning processes to be generated as models. This makes the model invalid. Synthetic Minority Over-sampling Technique (SMOTE) is a way to balance the dataset, if machine learning is done, in order to produce a good model.

4) The dependent variable and the independent variable are not clear or have no label. 


\section{Pipeline 3: Training and Testing Process}

This stage is conducting training on the malware dataset. Training using the KNN Algorithm, Support Vector Machine and Decision Tree. The distribution of the dataset is carried out, the training dataset is $70 \%$ and the testing dataset is $30 \%$. The Multi-Layer Perceptron Classifier algorithm is also used for this stage. The training process is also carried out using changes in the position of the training dataset and testing dataset, which is better known as cross validation. In this study using 5 -fold cross validation, to get better model accuracy.

Cross Validation (CV) is a method used to evaluate model performance, where data is separated into two subsets, namely learning process data and evaluation data. The model or algorithm is trained by the learning subset and validated by the validation subset. Furthermore, the selection of the type of $\mathrm{CV}$ can be based on the size of the dataset. CV K-fold is used because it can reduce computation time while maintaining the accuracy of the estimate. 5-fold $\mathrm{CV}$ is one of the $\mathrm{K}$-fold $\mathrm{CVs}$ used for selecting the best model because it tends to provide less biased accuracy estimates. In 5 -fold $\mathrm{CV}$, the dataset is divided into 5 folds of approximately equal size, thus having 5 subsets of data to evaluate model performance. For each of these 5 subsets of data, CV will use 4 folds for training data and 1-fold for testing.

\section{Pipeline 4: Prepare New APK data to be tested}

At this stage the aim is to add new datasets. If in performing the classification and new variants of malware are found, before being entered into the dataset, the data must be feature extraction. Then retraining is carried out. The more datasets, the better the classification model in identifying malware APK.

\section{E. Pipeline 5: Decision Classification Output Label}

The last stage aims to produce a classification model and the model is ready for deployment. Testing the model before the model is ready for use, aims to anticipate model errors in identifying Android APK files.

\section{EXPERIMENT AND RESULT}

In conducting the experiment, using the MacBook Air 2020 hardware with specifications of 8 GB RAM, 256 GB storage. Using the Python programming language in the Jupiter Notebook package, the reverse engineer JADX module made by APKTOOL. In this section, answer research questions and report experimental results.

\section{A. RQ 1, How to extract malware dataset using permission feature and intent feature?}

This is a much-needed step, where this step generates a malware dataset. APK files are downloaded and extracted, reverse engineered and parsed to read feature permissions and feature intents. The final result of feature extraction is a malware dataset. Following are the featurefeature permissions of the malware dataset:
TABLE I. Normal Feature Permission

\begin{tabular}{|c|c|}
\hline $\begin{array}{l}\text { ACCESS_ALL_DOWNLOAD } \\
\mathrm{S}\end{array}$ & $\begin{array}{l}\text { ACCESS_CACHE_FILESYST } \\
\text { EM }\end{array}$ \\
\hline BLUETOOTH_SHARE & $\begin{array}{l}\text { ACCESS_CHECKIN_PROPE } \\
\text { RTIES }\end{array}$ \\
\hline $\begin{array}{l}\text { ACCESS_DOWNLOAD_MA } \\
\text { NAGER_ADVANCED }\end{array}$ & $\begin{array}{l}\text { ACCESS_DRM_CERTIFICA } \\
\text { TES }\end{array}$ \\
\hline $\begin{array}{l}\text { ACCESS_NETWORK_COND } \\
\text { ITIONS }\end{array}$ & ACCESS_NOTIFICATIONS \\
\hline $\begin{array}{l}\text { ACCESS_NETWORK_STAT } \\
\text { E }\end{array}$ & $\begin{array}{l}\text { ACCESS_NOTIFICATION_P } \\
\text { OLICY }\end{array}$ \\
\hline ACCESS_PDB_STATE & BLUETOOTH_PRIVILEGED \\
\hline BIND_DEVICE_ADMIN & BIND_CARRIER_SERVICES \\
\hline $\begin{array}{l}\text { BIND_CARRIER_MESSAGI } \\
\text { NG_SERVICE }\end{array}$ & BIND_APPWIDGET \\
\hline $\begin{array}{l}\text { BIND_ACCESSIBILITY_SER } \\
\text { VICE }\end{array}$ & $\begin{array}{l}\text { BROADCAST_PHONE_ACC } \\
\text { OUNT_REGISTRATION }\end{array}$ \\
\hline BROADCAST_SMS & $\begin{array}{l}\text { BIND_ACCESSIBILITY_SER } \\
\text { VICE }\end{array}$ \\
\hline CAPTURE_AUDIO_OUTPUT & CALL_PRIVILEGED \\
\hline $\begin{array}{l}\text { CONFIGURE_WIFI_DISPLA } \\
\mathrm{Y}\end{array}$ & $\begin{array}{l}\text { CHANGE_CONFIGURATIO } \\
\mathrm{N}\end{array}$ \\
\hline $\begin{array}{l}\text { INSTALL_GRANT_RUNTIM } \\
\text { E_PERMISSIONS }\end{array}$ & $\begin{array}{l}\text { INTERNAL_SYSTEM_WIND } \\
\text { OW }\end{array}$ \\
\hline $\begin{array}{l}\text { INSTALL_LOCATION_PRO } \\
\text { VIDER }\end{array}$ & $\begin{array}{l}\text { INTERACT_ACROSS_USER } \\
\text { S_FULL }\end{array}$ \\
\hline GET_PASSWORD & $\begin{array}{l}\text { KILL_BACKGROUND_PRO } \\
\text { CESSES }\end{array}$ \\
\hline $\begin{array}{l}\text { INTERNAL_SYSTEM_WIND } \\
\text { OW }\end{array}$ & $\begin{array}{l}\text { INSTALL_GRANT_RUNTIM } \\
\text { E_PERMISSIONS }\end{array}$ \\
\hline MANAGE_DOCUMENTS & MANAGE_ACCOUNTS \\
\hline HARDWARE_TEST & $\begin{array}{l}\text { INSTALL_LOCATION_PRO } \\
\text { VIDER }\end{array}$ \\
\hline FORCE_STOP_PACKAGES & MANAGE_APP_TOKENS \\
\hline INSTALL_PACKAGES & KILL_UID \\
\hline
\end{tabular}

TABLE II. Normal Feature Intent

\begin{tabular}{|l|l|}
\hline $\begin{array}{l}\text { ACTION_POWER_CONNE } \\
\text { CTED }\end{array}$ & $\begin{array}{l}\text { ACTION_POWER_DISCONNE } \\
\text { CTED }\end{array}$ \\
\hline ACTION_SHUTDOWN & AIRPLANE_MODE \\
\hline BATTERY_CHANGED & BATTERY_LOW \\
\hline BATTERY_OKAY & BOOT_COMPLETED \\
\hline CAMERA_BUTTON & CONFIGURATION_CHANGED \\
\hline CREATE_SHORTCUT & DATE_CHANGED \\
\hline DEVICE_STORAGE_LOW & DEVICE_STORAGE_OK \\
\hline DOCK_EVENT & DREAMING_STARTED \\
\hline
\end{tabular}




\begin{tabular}{|l|l|}
\hline DREAMING_STOPPED & $\begin{array}{l}\text { EXTERNAL_APPLICATIONS_ } \\
\text { AVAILABLE }\end{array}$ \\
\hline $\begin{array}{l}\text { EXTERNAL_APPLICATIO } \\
\text { NS_UNAVAILABLE }\end{array}$ & FETCH_VOICEMAIL \\
\hline GTALK_CONNECTED & GTALK_DISCONNECTED \\
\hline HEADSET_PLUG & INPUT_METHOD_CHANGED \\
\hline LOCALE_CHANGED & $\begin{array}{l}\text { MANAGE_PACKAGE_STORA } \\
\text { GE }\end{array}$ \\
\hline MAIN & MEDIA_BAD_REMOVAL \\
\hline MEDIA_BUTTON & MEDIA_CHECKING \\
\hline MEDIA_MOUNTED & MEDIA_REMOVED \\
\hline PACKAGE_ADDED & PACKAGE_DATA_CLEARED \\
\hline PACKAGE_CHANGED & $\begin{array}{l}\text { MEDIA_SCANNER_SCAN_FIL } \\
\text { E }\end{array}$ \\
\hline PACKAGE_INSTALL & $\begin{array}{l}\text { PACKAGE_NEEDS_VERIFICA } \\
\text { TION }\end{array}$ \\
\hline PACKAGE_REMOVED & PROVIDER_CHANGED \\
\hline ACTION_TIME_CHANGED & SIM_STATE_CHANGED \\
\hline SENT_SMS_ACTION & $\begin{array}{l}\text { ACTION_EXTERNAL_APPLIC } \\
\text { ATIONS_AVAILABLE }\end{array}$ \\
\hline
\end{tabular}

TABLE III. ACCURACY FOR ALGORITHM MACHINE LEARNING

\begin{tabular}{|l|c|c|c|}
\hline \multirow{2}{*}{ Algorithm } & \multicolumn{3}{|c|}{ Accuracy } \\
\cline { 2 - 4 } & $\mathbf{6 0 0}$ APK & $\begin{array}{c}\mathbf{7 0 0 0} \\
\text { APK }\end{array}$ & $\begin{array}{c}\mathbf{1 4 1 7 0} \\
\text { APK }\end{array}$ \\
\hline K Nearest Neighbour (KNN) & $88 \%$ & $86.8 \%$ & $88 \%$ \\
\hline Decision Tree (DT) & $100 \%$ & $89 \%$ & $91,3 \%$ \\
\hline $\begin{array}{l}\text { Support Vector Machine } \\
\text { (SVM) }\end{array}$ & $97 \%$ & $90 \%$ & $91 \%$ \\
\hline
\end{tabular}

Precision is the ratio of a positive correct prediction compared to the overall positive predicted outcome. Precision answers the question "What percentage of Android APK files are Malware correct from the total dataset that Malware predicts?". Precision $=(\mathrm{TP}) /(\mathrm{TP}+$ FP). Precision can be seen in Table V.

TABLE IV. PRESSION FOR ALgorithm MACHINE LEARNING

\begin{tabular}{|l|c|c|c|}
\hline \multirow{2}{*}{ Algorithm } & \multicolumn{3}{|c|}{ Precision } \\
\cline { 2 - 4 } & $\begin{array}{c}\mathbf{6 0 0} \\
\text { APK }\end{array}$ & $\begin{array}{c}\mathbf{7 0 0 0} \\
\text { APK }\end{array}$ & $\begin{array}{c}\mathbf{1 4 1 7 0} \\
\text { APK }\end{array}$ \\
\hline K-Nearest Neighbour (KNN) & $88 \%$ & $85.6 \%$ & $88 \%$ \\
\hline Decision Tree (DT) & $100 \%$ & $89.4 \%$ & $91.8 \%$ \\
\hline Support Vector Machine (SVM) & $96.5 \%$ & $90 \%$ & $91.4 \%$ \\
\hline
\end{tabular}

F1 Score is a weighted comparison of the average precision and recall. F1 Score $=2 *$ (Recall * Precision) $/$ (Recall + Precision). F1-Score can be seen in Table VI.

TABLE V. F1-SCORE FOR ALGORITHM MACHINE LEARNING

\begin{tabular}{|l|c|c|c|}
\hline \multirow{2}{*}{ Algorithm } & \multicolumn{3}{|c|}{ F1-Score } \\
\cline { 2 - 4 } & $\begin{array}{c}\mathbf{6 0 0} \\
\text { APK }\end{array}$ & $\begin{array}{c}\mathbf{7 0 0 0} \\
\text { APK }\end{array}$ & $\begin{array}{c}\mathbf{1 4 1 7 0} \\
\text { APK }\end{array}$ \\
\hline K-Nearest Neighbour (KNN) & $88 \%$ & $85.2 \%$ & $88 \%$ \\
\hline Decision Tree (DT) & $100 \%$ & $88.6 \%$ & $91.2 \%$ \\
\hline Support Vector Machine (SVM) & $96.7 \%$ & $89.4 \%$ & $90.4 \%$ \\
\hline
\end{tabular}

Recall is the ratio of true positive predictions compared to the total number of true positive data. Recall answers the question "What percentage of Android APK files are predicted to be malware compared to all students who are actually malware". Recall $=(\mathrm{TP}) /(\mathrm{TP}+\mathrm{FN})$. Recall can be seen in Table VII.

TABLE VI. ReCAll For Algorithm Machine LEARning

B. $R Q 2$, What is the percentage of application of the $K$ -

Nearest Neighbor algorithm, Support Vector Machine algorithm and Decision Tree algorithm?

Definition

$\mathrm{TP}=$ True Positive.

$\mathrm{TN}=$ True Negative.

$\mathrm{FP}=$ False Positive.

$\mathrm{FN}=$ False Negative.

Accuracy is the ratio of correct predictions (positive and negative) to the entire dataset. Accuracy and answer the question "What percentage of Android APK files correctly predicted Malware and Benign from the entire dataset of Android APK files". Accuracy $=(\mathrm{TP}+\mathrm{TN}) /$ $(\mathrm{TP}+\mathrm{FP}+\mathrm{FN}+\mathrm{TN})$. Accuracy can be seen in table IV.

\begin{tabular}{|l|c|c|c|}
\hline \multicolumn{2}{|c|}{ Algorithm } & \multicolumn{3}{|c|}{ Recall } \\
\cline { 2 - 4 } & $\begin{array}{c}\mathbf{6 0 0} \\
\text { APK }\end{array}$ & $\begin{array}{c}\mathbf{7 0 0 0} \\
\text { APK }\end{array}$ & $\begin{array}{c}\mathbf{1 4 1 7 0} \\
\text { APK }\end{array}$ \\
\hline $\begin{array}{l}\text { K-Nearest Neighbour } \\
\text { (KNN) }\end{array}$ & $88 \%$ & $85.2 \%$ & $88 \%$ \\
\hline Decision Tree (DT) & $100 \%$ & $88.8 \%$ & $91.2 \%$ \\
\hline $\begin{array}{l}\text { Support Vector Machine } \\
\text { (SVM) }\end{array}$ & $97 \%$ & 89.4 & $90.6 \%$ \\
\hline
\end{tabular}

There is a decrease in performance for the model generated from the K-Nearest Neighbor algorithm, Support Vector Machine and Decision Tree. Table IV, Accuracy of KNN, Support Vector and Decision Tree classifier decreased when using a larger dataset. This is because the three algorithms are suitable for use if the dataset is small. The larger the size of the training dataset, the lower the accuracy. Table V Precision decreased if the classifier was 
carried out with the three non-neural network algorithms. Table VI F1-Score experienced a decrease in the classification of large datasets and Table VII Recall decreases if the dataset is large. In this study, the use of a large dataset is not suitable when using a large dataset, and it is tried to train the dataset using a Neural Network.

\section{C. $R Q 3$, What is the percent increase in accuracy with the implementation of the Multi-Layer Perceptron algorithm?}

TABLE VII. PERFormance FOR Algorithm DEEP LEARNING

\begin{tabular}{|l|c|c|c|}
\hline \multirow{2}{*}{ Performance } & \multicolumn{3}{|c|}{ Dataset } \\
\cline { 2 - 4 } & $\begin{array}{c}\mathbf{6 0 0} \\
\text { APK }\end{array}$ & $\mathbf{7 0 0 0}$ APK & $\begin{array}{c}\mathbf{1 4 1 7 0} \\
\text { APK }\end{array}$ \\
\hline Accuracy & $99 \%$ & $100 \%$ & $100 \%$ \\
\hline Precision & $99 \%$ & $100 \%$ & $100 \%$ \\
\hline Recall & $99 \%$ & $100 \%$ & $100 \%$ \\
\hline F1-Score & $99 \%$ & $100 \%$ & $100 \%$ \\
\hline
\end{tabular}

The results of the Multi-Layer Perceptron classification experiment show that performance increases with increasing datasets. The more the number of datasets, the better for performance. Experiment from dataset 600 APK $=99 \%$, dataset 7000 APK $=100 \%$ and dataset 14170 APK $=100 \%$.

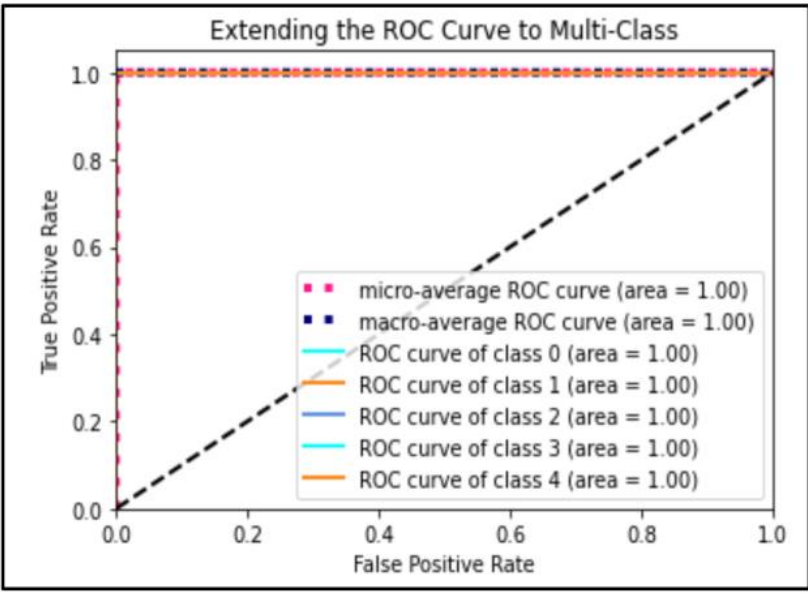

Fig. 4. ROC Multi-Class Multi-Layer Perceptron Algorithm

Receiver Operating Characteristic (ROC) is a plot of True Positive Rate (TPR) on the y-axis and False Positive Rate (FPR) on the $\mathrm{x}$-axis. Where, True Positive Rate $=$ True Positives / (True Positives + False Negatives) and False Positive Rate $=$ False Positives $/$ (False Positives + True Negatives). It can be seen that the ROC and Area Under Curve scores show significant values.

In Figure 4, the ROC of the model results from the Artificial Neural Network Classifier from the malware dataset. ROC (Receiver Operating Characteristics) is a performance measurement tool for classification problems in determining the threshold of the model. Malware Banking APK APK file label 0, symbolized in light blue. APK file Malware Ransomware APK label 1, symbolized in orange. APK file Malware Riskware APK label 2, symbolized in blue. Malware SMS APK file APK label 3, symbolized in light blue. APK file Malware Benign APK label 4, symbolized in orange. The y-axis represents the True Positive Rate (sensitivity), the $\mathrm{x}$-axis represents the False Positive Rate (Specificity). Figure 4 shows the higher the True Positive Rate (sensitivity) and the smaller the False Positive Rate, the better the threshold. The optimistic Area Under Curve (AUC) value from the Artificial Neural Network validation results shows a value of $=1$. This shows that the accuracy results obtained are in the very good category.

\section{D. $R Q 4$, Is it effective to perform malware analysis using static methods?}

Using this static method does not require running the malware into an isolated or controlled environment. The malware APK file is only extracted, then stored into the malware dataset. The dataset is classified using the classification method and then the model is tested with the extracted malware dataset. The results are effective for detecting the Android APK file is infected with malware or normal. The static method is actually simple and works effectively in malware detection.

\section{CONCLUTION}

Based on the results of experiments conducted in this study, it can be concluded that classification using machine learning produces good accuracy on the K-Nearest Neighbor algorithm, Support Vector Machine and Decision Tree. However, the use of larger datasets causes a decrease in accuracy. This factor causes the use of deep learning in training datasets in order to produce high accuracy on large datasets. The accuracy of the K-Nearest Neighbor algorithm on average $=88 \%$, if using the 14170 APK dataset. Average Support Vector Machine accuracy = $90.5 \%$, when using the 14170 APK dataset. Average Decision Tree accuracy $=90.8 \%$, when using the 14170 APK dataset. Accuracy using deep learning with MultiLayer Perceptron results in $100 \%$ accuracy, using the 14170 APK dataset.

\section{REFERENCES}

A. Ghasempour, N. Fazlida, M. Sani, and O. J. Abari, "Permission Extraction Framework for Android Malware Detection," vol. 11, no. 11, pp. 463-475, 2020. O. N. Elayan and A. M. Mustafa, "Android malware detection using deep learning," Procedia Comput. Sci., vol. 184, no. 2019, pp. 847-852, 2021, doi: 10.1016/j.procs.2021.03.106.

[3] S. R. T. Mat, M. F. A. Razak, M. N. M. Kahar, J. M. Arif, and A. Firdaus, "A Bayesian probability model for Android malware detection," ICT Express, no. xxxx, 2021, doi: 10.1016/j.icte.2021.09.003.

[4] M. Qiao, A. H. Sung, and Q. Liu, "Merging permission and api features for android malware detection," Proc. 2016 5th IIAI Int. Congr. Adv. Appl. Informatics, IIAIAAI 2016, pp. 566-571, 2016, doi: 10.1109/IIAIAAI.2016.237.

[5] A. K. Singh, C. D. Jaidhar, and M. A. A. Kumara, "Experimental analysis of Android malware detection based on combinations of permissions and API-calls," 
J. Comput. Virol. Hacking Tech., vol. 15, no. 3, pp. 209 218, 2019, doi: 10.1007/s11416-019-00332-z.

[6] M. Shohel Rana, C. Gudla, and A. H. Sung, "Evaluating machine learning models for android malware detection - A comparison study," ACM Int. Conf. Proceeding Ser., pp. 17-21, 2018, doi: 10.1145/3301326.3301390.

[7] X. Wang, D. Zhang, X. Su, and W. Li, "Mlifdect: Android malware detection based on parallel machine learning and information fusion," Secur. Commun. Networks, vol. 2017, 2017, doi: 10.1155/2017/6451260.

[8] H. Fereidooni, M. Conti, D. Yao, and A. Sperduti, "ANASTASIA: ANdroid mAlware detection using STatic analySIs of applications," 2016 8th IFIP Int. Conf. New Technol. Mobil. Secur. NTMS 2016, 2016, doi: 10.1109/NTMS.2016.7792435.

[9] A. Feizollah, N. B. Anuar, R. Salleh, G. Suarez-Tangil, and S. Furnell, "AndroDialysis: Analysis of Android Intent Effectiveness in Malware Detection," Comput. Secur., vol. 65, no. March, pp. 121-134, 2017, doi: 10.1016/j.cose.2016.11.007.

[10] F. Idrees, M. Rajarajan, M. Conti, T. M. Chen, and Y. Rahulamathavan, "PIndroid: A novel Android malware detection system using ensemble learning methods," Comput. Secur., vol. 68, pp. 36-46, 2017, doi: 10.1016/j.cose.2017.03.011.

[11] A. Abusitta, M. Q. Li, and B. C. M. Fung, "Malware classification and composition analysis: A survey of recent developments," J. Inf. Secur. Appl., vol. 59, no. April, p. 102828, 2021, doi: 10.1016/j.jisa.2021.102828.

[12] V. Syrris and D. Geneiatakis, "On machine learning effectiveness for malware detection in Android OS using static analysis data," J. Inf. Secur. Appl., vol. 59, no. May, p. 102794, 2021, doi: 10.1016/j.jisa.2021.102794.

[13] P. Opěla, I. Schindler, P. Kawulok, R. Kawulok, S. Rusz, and H. Navrátil, "On various multi-layer perceptron and radial basis function based artificial neural networks in the process of a hot flow curve description," J. Mater. Res. Technol., vol. 14, pp. 1837 1847, 2021, doi: 10.1016/j.jmrt.2021.07.100.

[14] M. L. Baptista, E. M. Elsa, and K. Goebel, "A selforganizing map and a normalizing multi-layer perceptron approach to baselining in prognostics under dynamic regimes," Neurocomputing, vol. 456, pp. 268 287, 2021, doi: 10.1016/j.neucom.2021.05.031.

[15] M. Amin, T. A. Tanveer, M. Tehseen, M. . Khan, F. A Khan, and S. Anwar, "Static malware detection and attribution in android byte-code through an end-to-end deep system," Futur. Gener. Comput. Syst., vol. 102, pp. 112-126, 2020, doi: 10.1016/j.future.2019.07.070.

[16] L. Xiong and Y. Yao, "Study on an adaptive thermal comfort model with K-nearest-neighbors (KNN) algorithm," Build. Environ., vol. 202, no. May, p. 108026, 2021, doi: 10.1016/j.buildenv.2021.108026.

[17] D. Sisodia and D. S. Sisodia, "Quad division prototype selection-based k-nearest neighbor classifier for click fraud detection from highly skewed user click dataset," Eng. Sci. Technol. an Int. J., no. xxxx, 2021, doi: 10.1016/j.jestch.2021.05.015.

[18] A. Shokrzade, M. Ramezani, F. Akhlaghian Tab, and M.

[31] J. Mariéthoz and S. Bengio, "A kernel trick for sequences applied to text-independent speaker verification systems," Pattern Recognit., vol. 40, no. 8, pp. 2315-2324, 2007, doi
Abdulla Mohammad, "A novel extreme learning machine based $\mathrm{kNN}$ classification method for dealing with big data," Expert Syst. Appl., vol. 183, no. May, p. 115293, 2021, doi: 10.1016/j.eswa.2021.115293.

[19] X. Zhu, C. Ying, J. Wang, J. Li, X. Lai, and G. Wang, "Ensemble of ML-KNN for classification algorithm recommendation," Knowledge-Based Syst., vol. 221, p. 106933, 2021, doi: 10.1016/j.knosys.2021.106933.

[20] C. E. A. Bundak, M. A. Abd Rahman, M. K. Abdul Karim, and N. H. Osman, "Fuzzy rank cluster top k Euclidean distance and triangle based algorithm for magnetic field indoor positioning system," Alexandria Eng. J., 2021, doi: 10.1016/j.aej.2021.08.073.

[21] R. Taheri, M. Ghahramani, R. Javidan, M. Shojafar, Z. Pooranian, and M. Conti, "Similarity-based Android malware detection using Hamming distance of static binary features," Futur. Gener. Comput. Syst., vol. 105, pp. 230-247, 2020, doi: 10.1016/j.future.2019.11.034.

[22] R. Suwanda, Z. Syahputra, and E. M. Zamzami, "Analysis of Euclidean Distance and Manhattan Distance in the K-Means Algorithm for Variations Number of Centroid K," J. Phys. Conf. Ser., vol. 1566, no. 1, 2020, doi: 10.1088/1742-6596/1566/1/012058.

[23] S. Zhang, "Cost-sensitive KNN classification," Neurocomputing, vol. 391, no. xxxx, pp. 234-242, 2020, doi: 10.1016/j.neucom.2018.11.101.

[24] T. Du, Z. Zhao, Q. Zhu, and L. Tian, "Locating a $\gamma$-ray source using cuboid scintillators and the KNN algorithm," Nucl. Instruments Methods Phys. Res. Sect. A Accel. Spectrometers, Detect. Assoc. Equip., vol. 993, no. January, 2021, doi: 10.1016/j.nima.2021.165069.

[25] R. M. Arias Velásquez, "Support vector machine and tree models for oil and Kraft degradation in power transformers," Eng. Fail. Anal., vol. 127, no. May, 2021, doi: 10.1016/j.engfailanal.2021.105488.

[26] Y. Arbabi Yazdi, H. Toossian Shandiz, and H. Gholizadeh Narm, "Stiction detection in control valves using a support vector machine with a generalized statistical variable," ISA Trans., no. xxxx, 2021, doi: 10.1016/j.isatra.2021.07.020.

[27] J. Lesouple, C. Baudoin, M. Spigai, and J. Y. Tourneret, "How to introduce expert feedback in one-class support vector machines for anomaly detection?," Signal Processing, vol. 188, p. 108197, 2021, doi: 10.1016/j.sigpro.2021.108197.

[28] X. Ju, Y. Tian, D. Liu, and Z. Qi, "Nonparallel hyperplanes support vector machine for multi-class classification," Procedia Comput. Sci., vol. 51, no. 1, pp. 1574-1582, 2015, doi 10.1016/j.procs.2015.05.287.

[29] Q. Zhang, H. Wang, and S. W. Yoon, "A 1-norm regularized linear programming nonparallel hyperplane support vector machine for binary classification problems," Neurocomputing, vol. 376, no. xxxx, pp. 141-152, 2020, doi: 10.1016/j.neucom.2019.09.068.

[30] Z. Zhao, P. Zhong, and Y. Zhao, "Learning SVM with weighted maximum margin criterion for classification of imbalanced data," Math. Comput. Model., vol. 54, no. 3-4, pp. 1093-1099, 2011, doi: 10.1016/j.mcm.2010.11.040.

10.1016/j.patcog.2007.01.011.

[32] S. F. Hussain, "A novel robust kernel for classifying high-dimensional data using Support Vector Machines," Expert Syst. Appl., vol. 131, pp. 116-131, 2019, doi: 
10.1016/j.eswa.2019.04.037.

[33] X. Huang, A. Maier, J. Hornegger, and J. A. K. Suykens, "Indefinite kernels in least squares support vector machines and principal component analysis," Appl. Comput. Harmon. Anal., vol. 43, no. 1, pp. 162-172, 2017, doi: 10.1016/j.acha.2016.09.001.

[34] M. Moshkov, "Decision trees based on 1consequences," Discret. Appl. Math., vol. 302, pp. 208 214, 2021, doi: 10.1016/j.dam.2021.07.017.

[35] V. Gumuskaya, W. van Jaarsveld, R. Dijkman, P. Grefen, and A. Veenstra, "Integrating stochastic programs and decision trees in capacitated barge planning with uncertain container arrivals," Transp. Res. Part C Emerg. Technol., vol. 132, no. December 2020, p. 103383, 2021, doi: 10.1016/j.trc.2021.103383.

[36] A. Strzelecka and D. Zawadzka, "Application of classification and regression tree (CRT) analysis to identify the agricultural households at risk of financial exclusion," Procedia Comput. Sci., vol. 192, pp. 45324541, 2021, doi: 10.1016/j.procs.2021.09.231.

[37] D. H. Lee, S. H. Kim, and K. J. Kim, "Multistage MRCART: Multiresponse optimization in a multistage process using a classification and regression tree method," Comput. Ind. Eng., vol. 159, no. May, p. 107513, 2021, doi: 10.1016/j.cie.2021.107513.

[38] M. Li, P. Vanberkel, and X. Zhong, "Predicting ambulance offload delay using a hybrid decision tree model," Socioecon. Plann. Sci., no. July, p. 101146, 2021, doi: 10.1016/j.seps.2021.101146.

[39] W. Gao, Z. Bai, F. Zhu, C. C. Chou, and B. Jiang, "A study on the cyclist head kinematic responses in electric-bicycle-to-car accidents using decision-tree model," Accid. Anal. Prev., vol. 160, no. May 2020, p. 106305, 2021, doi: 10.1016/j.aap.2021.106305. 